\title{
^-HYPERCONNECTED IDEAL TOPOLOGICAL SPACES
}

BY

\author{
ERDAL EKICI and TAKASHI NOIRI
}

\begin{abstract}
The aim of this paper is to introduce and study $\star$-hyperconnected ideal topological spaces. Characterizations and properties of $\star$-hyperconnected ideal topological spaces are investigated.

Mathematics Subject Classification 2000: 54A05, 54A10.

Key words: $\star$-hyperconnected ideal topological space, $\star$-nowhere dense set, $\star$-dense set, semi*-I-open set.

\section{Introduction}

Several notions which are equivalent to hyperconnectedness were investigated in the literature such as the notions of $D$-spaces, semi-connected spaces, s-connected spaces, irreducible spaces. On the other hand, the notion of ideal topological spaces was studied by KuRATOWsKI [7] and Vaidyanathaswamy [9]. In 1990, Janković and Hamlett [6] investigated further properties of ideal topological spaces. In this paper, the notion of $\star$-hyperconnected ideal topological spaces are introduced and studied. Characterizations and properties of $\star$-hyperconnected ideal topological spaces are investigated.
\end{abstract}

\section{Preliminaries}

Throughout the present paper, $(X, \tau)$ or $(Y, \sigma)$ will denote a topological space with no separation properties assumed. For a subset $A$ of a topological space $(X, \tau), C l(A)$ and $\operatorname{Int}(A)$ will denote the closure and interior of $A$ in $(X, \tau)$, respectively. An ideal $I$ on a topological space $(X, \tau)$ is a nonempty collection of subsets of $X$ which satisfies (1) $A \in I$ and $B \subset A$ implies 
$B \in I,(2) A \in I$ and $B \in I$ implies $A \cup B \in I$. Given a topological space $(X, \tau)$ with an ideal $I$ on $X$ and, if $P(X)$ is the set of all subsets of $X$, a set operator (.)* $: P(X) \rightarrow P(X)$, called a local function ([7]) of $A$ with respect to $\tau$ and $I$ is defined as follows: for $A \subset X, A^{*}(I, \tau)=\{x \in X: G \cap A \notin I$ for every $G \in \tau(x)\}$ where $\tau(x)=\{G \in \tau: x \in G\}$. A Kuratowski closure operator $C l^{*}($.$) for a topology \tau^{*}(I, \tau)$, called the $\star$-topology, finer than $\tau$, is defined by $C l^{*}(A)=A \cup A^{*}(I, \tau)([6])$. When there is no chance for confusion, we will simply write $A^{*}$ for $A^{*}(I, \tau)$ and $\tau^{*}$ or $\tau^{*}(I)$ for $\tau^{*}(I, \tau)$. For any ideal space $(X, \tau, I)$, the collection $\{U \backslash J: U \in \tau$ and $J \in I\}$ is a basis for $\tau^{*}$. If $I$ is an ideal on $X$, then $(X, \tau, I)$ is called an ideal topological space or simply an ideal space.

Definition 1. A subset $A$ of an ideal space $(X, \tau, I)$ is said to be:

(1) pre- $I$-open ([1]) if $A \subset \operatorname{Int}\left(C l^{*}(A)\right)$;

(2) semi- $I$-open ([3]) if $A \subset C l^{*}(\operatorname{Int}(A))$;

(3) strongly $\beta$-I-open ([4]) if $A \subset C l^{*}\left(\operatorname{Int}\left(C l^{*}(A)\right)\right)$;

(4) $\star$-dense ([2]) if $C l^{*}(A)=X$;

(5) $\star$-nowhere dense if $\operatorname{Int}\left(C l^{*}(A)\right)=\emptyset$.

The complement of a pre- $I$-open (resp. semi- $I$-open, strongly $\beta$ - $I$-open) set is called pre- $I$-closed (resp. semi- $I$-closed, strongly $\beta$ - $I$-closed). A topological space $X$ is said to be hyperconnected ([8]) if every pair of nonempty open sets of $X$ has nonempty intersection. A function $f:(X, \tau, I) \rightarrow(Y, \sigma)$ is said to be semi- $I$-continuous ([3]) if, for every open set $A$ of $Y, f^{-1}(A)$ is semi- $I$-open in $X$.

\section{Characterizations of $\star$-hyperconnected spaces}

Definition 2. An ideal space $(X, \tau, I)$ is said to be:

(1) $\star$-hyperconnected if $A$ is $\star$-dense for every open subset $A \neq \emptyset$ of $X$;

(2) $\star$-connected if $X$ can not be written as the union of nonempty and disjoint an open set and a $\star$-open set of $X$. 
Remark 3. (1) Generally, it is known that every hyperconnected topological space is connected, but not conversely.

(2) For an ideal space $(X, \tau, I), \tau \subset \tau^{*}$ and we have the following properties:

$$
\begin{array}{ccc}
(X, \tau, I) \text { is } \star \text {-hyperconnected } & \Rightarrow(X, \tau) \text { is hyperconnected } \\
\Downarrow & \Downarrow \\
(X, \tau, I) \text { is } \star \text {-connected } & \Rightarrow \quad(X, \tau) \text { is connected }
\end{array}
$$

The implications in the diagram are not reversible as shown in the following examples:

Example 4. Let $X=\{a, b, c\}, \tau=\{X, \emptyset,\{a\},\{a, b\},\{a, c\}\}$ and $I=$ $\{\emptyset,\{a\}\}$. Then the space $(X, \tau)$ is hyperconnected but $(X, \tau, I)$ is not $\star-$ connected.

Example 5. Let $X=\{a, b, c, d\}, \tau=\{X, \emptyset,\{a\},\{c\},\{a, b\},\{a, c\}$, $\{a, b, c\},\{a, c, d\}\}$ and $I=\{\emptyset,\{b\}\}$. Then, the ideal space $(X, \tau, I)$ is $\star$-connected but it is not hyperconnected.

Definition 6. A subset $A$ of an ideal space $(X, \tau, I)$ is called

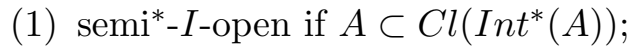

(2) semi*-I-closed if its complement is semi*-I-open.

Lemma 7. Every semi-I-open set is semi*-I-open.

Proof. Let $A$ be a semi- $I$-open subset in an ideal space $(X, \tau, I)$. Then $A \subset C l^{*}(\operatorname{Int}(A))$. We have $A \subset C l^{*}(\operatorname{Int}(A)) \subset C l\left(\operatorname{Int} t^{*}(A)\right)$. Thus, $A$ is semi*-I-open.

The implication in Lemma 7 is not reversible as shown in the following example:

Example 8. Let $X=\{a, b, c, d\}, \tau=\{X, \emptyset,\{a\},\{a, b\},\{c, d\},\{a, c, d\}\}$ and $I=\{\emptyset,\{a\},\{d\},\{a, d\}\}$. Then, the set $A=\{b, c, d\}$ is semi*-I-open but it is not semi- $I$-open.

Lemma 9 ([5]). A subset $A$ of an ideal space $(X, \tau, I)$ is semi-I-open, if and only if there exists $B \in \tau$, such that $B \subset A \subset C l^{*}(B)$.

Lemma 10. A subset $A$ of an ideal space $(X, \tau, I)$ is semi*-I-open, if and only if there exists $B \in \tau^{*}$, such that $B \subset A \subset C l(B)$. 
Proof. Let $A$ be semi*-I-open. Then $A \subset C l\left(\operatorname{Int} t^{*}(A)\right)$. Take $B=$ Int $^{*}(A)$. We have $B \subset A \subset C l(B)$.

Conversely, let $B \subset A \subset C l(B)$ for a $B \in \tau^{*}$. Since $B \subset A$, then $B \subset \operatorname{Int}^{*}(A)$. Thus, $C l(B) \subset C l\left(\operatorname{Int}^{*}(A)\right)$ and $A \subset C l\left(\operatorname{Int}^{*}(A)\right)$. Hence, $A$ is semi*- $I$-open.

Theorem 11. Let $(X, \tau, I)$ be an ideal space. The following properties are equivalent:

(1) $X$ is $\star$-hyperconnected;

(2) $A$ is $\star$-dense or $\star$-nowhere dense, for every subset $A \subset X$;

(3) $A \cap B \neq \emptyset$, for every nonempty open subset $A$ and every nonempty $\star$-open subset $B$ of $X$;

(4) $A \cap B \neq \emptyset$, for every nonempty semi-I-open subset $A \subset X$ and every nonempty semi*-I-open subset $B \subset X$.

Proof. $(1) \Rightarrow(2)$ : Let $X$ be $\star$-hyperconnected and $A \subset X$. Suppose that $A$ is not $\star$-nowhere dense. Then $C l\left(X \backslash C l^{*}(A)\right)=X \backslash \operatorname{Int}\left(C l^{*}(A)\right) \neq$ $X$. By (1), for $\operatorname{Int}\left(C l^{*}(A)\right) \neq \emptyset, C l^{*}\left(\operatorname{Int}\left(C l^{*}(A)\right)\right)=X$.

Since $C l^{*}\left(\operatorname{Int}\left(C l^{*}(A)\right)\right)=X \subset C l^{*}(A)$, then $C l^{*}(A)=X$. Thus, $A$ is $\star$-dense.

$(2) \Rightarrow(3)$ : Suppose that $A \cap B=\emptyset$, for some nonempty sets $A \in \tau$ and $B \in \tau^{*}$. Then $C l^{*}(A) \cap B=\emptyset$ and $A$ is not $\star$-dense. Moreover, since $A \in \tau$, $\emptyset \neq A \subset \operatorname{Int}\left(C l^{*}(A)\right)$ and $A$ is not $\star$-nowhere dense.

$(3) \Rightarrow(4)$ : Suppose that $A \cap B=\emptyset$, for some nonempty semi- $I$-open set $A$ and some nonempty semi*- $I$-open set $B$. By Lemmas 9 and 10 , there exist $M \in \tau$ and $N \in \tau^{*}$, such that $M \subset A \subset C l^{*}(M)$ and $N \subset B \subset C l(N)$. Since $A$ and $B$ are nonempty, $M$ and $N$ are nonempty. Moreover, we have $M \cap N \subset A \cap B=\emptyset$.

(4) $\Rightarrow(1)$ : Suppose that $A \cap B \neq \emptyset$, for every nonempty semi- $I$-open subset $A \subset X$ and every nonempty semi*-I-open subset $B \subset X$. Since every open set is semi- $I$-open and every $\star$-open set is semi*- $I$-open, then $X$ is $\star$-hyperconnected.

Definition 12. The semi*- $I$-closure (resp. semi- $I$-closure, pre- $I$-closure, strongly $\beta$-I-closure) of a subset $A$ of an ideal space $(X, \tau, I)$, denoted by $S^{*}-I-C l(A)$ (resp. $S-I-C l(A), P-I-C l(A), S \beta-I-C l(A)$ ), is defined by the intersection of all semi* ${ }^{*} I$-closed (resp. semi- $I$-closed, pre- $I$-closed, strongly $\beta$ - $I$-closed) sets of $X$ containing $A$. 
Lemma 13. The following properties hold for a subset $A$ of an ideal space $(X, \tau, I)$ :

(1) $S^{*}-I-C l(A)=A \cup \operatorname{Int}\left(C l^{*}(A)\right)$;

(2) $S-I-C l(A)=A \cup \operatorname{Int}^{*}(C l(A))$;

(3) $P-I-C l(A)=A \cup C l\left(\operatorname{Int}^{*}(A)\right)$;

(4) $S \beta-I-C l(A)=A \cup \operatorname{Int} t^{*}\left(C l\left(\operatorname{Int}^{*}(A)\right)\right)$.

Proof. (4) Since $S \beta-I-C l(A)$ is strongly $\beta$ - $I$-closed, then

$$
\operatorname{Int}^{*}\left(C l\left(\operatorname{Int}^{*}(A)\right)\right) \subset \operatorname{Int}^{*}\left(C l\left(\operatorname{Int}^{*}(S \beta-I-C l(A))\right)\right) \subset S \beta-I-C l(A) .
$$

Hence, $A \cup \operatorname{Int} t^{*}(C l(\operatorname{Int}(A))) \subset S \beta-I-C l(A)$. Conversely, we have:

$$
\begin{aligned}
& \operatorname{Int}^{*}\left(C l\left(\operatorname{Int}^{*}\left(A \cup \operatorname{Int} t^{*}\left(C l\left(\operatorname{Int}^{*}(A)\right)\right)\right)\right)\right) \\
& \subset \operatorname{Int}^{*}\left(C l\left(\operatorname{Int}^{*}\left(A \cup C l\left(\operatorname{Int}^{*}(A)\right)\right)\right)\right) \\
& \left.\subset \operatorname{Int}^{*}\left(C l\left(\operatorname{Int}^{*}(A) \cup C l\left(\operatorname{Int}^{*}(A)\right)\right)\right)\right) \\
& \left.=\operatorname{Int}^{*}\left(C l\left(\operatorname{Int}^{*}(A)\right)\right) \subset A \cup \operatorname{Int}^{*}\left(C l\left(\operatorname{Int}^{*}(A)\right)\right)\right) .
\end{aligned}
$$

Then $A \cup \operatorname{Int^{*}}\left(C l\left(\operatorname{Int} t^{*}(A)\right)\right)$ is strongly $\beta$-I-closed containing $A$. Thus, $S \beta-I-C l(A) \subset A \cup \operatorname{Int} t^{*}\left(C l\left(\operatorname{Int} t^{*}(A)\right)\right)$. Hence,

$$
S \beta-I-C l(A)=A \cup \operatorname{Int}^{*}\left(C l\left(\operatorname{Int}^{*}(A)\right)\right) .
$$

The proofs of (1), (2) and (3) are similar to that of (4).

Theorem 14. The following are equivalent for an ideal space $(X, \tau, I)$ :

(1) $X$ is $\star$-hyperconnected;

(2) $H$ is $\star$-dense, for every strongly $\beta$-I-open subset $\emptyset \neq H \subset X$;

(3) $S^{*}-I-C l(H)=X$, for every strongly $\beta$-I-open subset $\emptyset \neq H \subset X$;

(4) $S \beta-I-C l(G)=X$, for every semi $i^{*} I$-open subset $\emptyset \neq G \subset X$;

(5) $P$-I-Cl $(G)=X$, for every semi* $i^{*}$-open subset $\emptyset \neq G \subset X$. 
Proof. $(1) \Rightarrow(2)$ : Let $(X, \tau, I)$ be a $\star$-hyperconnected ideal space. Let $H$ be any nonempty strongly $\beta$ - $I$-open subset of $X$. We have $\operatorname{Int}\left(C l^{*}(H)\right) \neq$ $\emptyset$. Thus, $X=C l^{*}\left(\operatorname{Int}\left(C l^{*}(H)\right)\right)=C l^{*}(H)$.

$(2) \Rightarrow(3)$ : Let $H$ be any nonempty strongly $\beta$ - $I$-open subset of $X$. Thus, by Lemma 13, $S^{*}-I-C l(H)=H \cup \operatorname{Int}\left(C l^{*}(H)\right)=H \cup \operatorname{Int}(X)=X$.

$(3) \Rightarrow(4)$ : Let $G$ be a nonempty semi*-I-open subset of $X$. Thus, by Lemma 13,

$$
\begin{aligned}
S \beta-I-C l(G) & =G \cup \operatorname{Int} *^{*}\left(C l\left(\operatorname{Int}^{*}(G)\right)\right)=G \cup \operatorname{Int}^{*}(C l(G)) \\
& \supset G \cup \operatorname{Int}\left(C l^{*}(G)\right) \supset \operatorname{Int}(G) \cup \operatorname{Int}\left(C l^{*}(\operatorname{Int}(G))\right) \\
& =S^{*}-I-C l(\operatorname{Int}(G))=X .
\end{aligned}
$$

Thus, $S \beta-I-C l(G)=X$.

$(4) \Rightarrow(5)$ : Let $G$ be a nonempty semi*- $I$-open subset of $X$. By Lemma 13, we have $P-I-C l(G)=G \cup C l\left(\operatorname{Int}^{*}(G)\right) \supset G \cup \operatorname{Int}^{*}\left(C l\left(\operatorname{Int}^{*}(G)\right)\right)=$ $S \beta-I-C l(G)=X$. Thus, $P-I-C l(G)=X$.

$(5) \Rightarrow(1)$ : Let $G$ be a nonempty $\star$-open set of $X$. By $(5), P-I-C l(G)=$ $G \cup C l\left(\operatorname{Int}^{*}(G)\right)=X$. This implies that $C l(G)=X$. By Theorem $11, X$ is $\star$-hyperconnected.

Corollary 15. For an ideal space $(X, \tau, I)$, the following properties are equivalent:

(1) $X$ is «-hyperconnected;

(2) $G \cap H \neq \emptyset$, for every nonempty semi*-I-open subset $G \subset X$ and every nonempty strongly $\beta$-I-open subset $H \subset X$;

(3) $G \cap H \neq \emptyset$, for any nonempty semi* $-I$-open set $G$ and any nonempty pre-I-open set $H$.

Proof. The proof is obvious from Theorem 14 .

\section{4. $\star$-hyperconnected spaces and functions}

Definition 16. The semi- $I$-interior of a subset $A$ of an ideal space $(X, \tau, I)$, denoted by $S$ - $I$ - $\operatorname{Int}(A)$, is defined by the union of all semi- $I$-open sets of $X$ contained in $A$. 
Definition 17. A function $f:(X, \tau, I) \rightarrow(Y, \sigma)$ is said to be almost $F-I$-continuous if for every nonempty regular open set $A$ of $Y, f^{-1}(A) \neq \emptyset$ implies $S$-I-Int $\left(f^{-1}(A)\right) \neq \emptyset$.

Theorem 18. Every semi-I-continuous function $f:(X, \tau, I) \rightarrow(Y, \sigma)$ is almost $F$-I-continuous.

Proof. Let $f:(X, \tau, I) \rightarrow(Y, \sigma)$ be a semi- $I$-continuous function. Let $A$ be any regular open subset of $Y$ such that $f^{-1}(A) \neq \emptyset$. Then $f^{-1}(A)$ is a nonempty semi- $I$-open set in $X$ and hence, $f^{-1}(A)=S$-I-Int $\left(f^{-1}(A)\right)$. Thus, $f$ is almost $F$ - $I$-continuous.

The implication in Theorem 18 is not reversible as shown in the following example:

Example 19. Let $X=Y=\{a, b, c, d\}, \tau=\sigma=\{X, \emptyset,\{a\},\{a, b\},\{c, d\}$, $\{a, c, d\}\}$ and $I=\{\emptyset,\{a\},\{d\},\{a, d\}\}$. Define the function $f:(X, \tau, I) \rightarrow$ $(Y, \sigma)$ as follows: $f(a)=a, f(b)=c, f(c)=d, f(d)=c$. Then $f$ is almost $F$ - $I$-continuous but it is not semi- $I$-continuous.

Theorem 20. The following properties hold for $a \star$-hyperconnected ideal space $(X, \tau, I)$ :

(1) Every almost $F$-I-continuous function $f:(X, \tau, I) \rightarrow(Y, \sigma)$, where $(Y, \sigma)$ is a Hausdorff space is constant;

(2) Every semi-I-continuous function $f:(X, \tau, I) \rightarrow(Y, \sigma)$, where $(Y, \sigma)$ is a Hausdorff space is constant;

(3) Every semi-I-continuous function $f:(X, \tau, I) \rightarrow(Y, \sigma)$, where $(Y, \sigma)$ is a two point discrete space is constant.

Proof. (1) : Let $X$ be a $\star$-hyperconnected ideal space. Suppose that there exist a Hausdorff space $Y$ and an almost $F-I$-continuous function $f: X \rightarrow Y$, such that $f$ is not constant. There exist two points $x$ and $y$ of $X$, such that $f(x) \neq f(y)$. Since $Y$ is Hausdorff, then there exist open sets $A$ and $B$ in $Y$, such that $f(x) \in A, f(y) \in B$ and $A \cap B=\emptyset$. Take $M=\operatorname{Int}(C l(A))$ and $N=\operatorname{Int}(C l(B))$. This implies that $M$ and $N$ are nonempty regular open and $M \cap N=\emptyset$. Since $f$ is almost $F-I$ continuous, then $S-I-\operatorname{Int}\left(f^{-1}(M)\right) \neq \emptyset$ and $S-I-\operatorname{Int}\left(f^{-1}(N)\right) \neq \emptyset$. We have $S$-I-Int $\left(f^{-1}(M)\right) \cap S$-I-Int $\left(f^{-1}(N)\right) \subset f^{-1}(M \cap N)=\emptyset$. Since $S$ - I$\operatorname{Int}\left(f^{-1}(M)\right)$ and $S-I-\operatorname{Int}\left(f^{-1}(N)\right)$ are semi- $I$-open, then by Lemma 7 and Theorem $11, X$ is not $\star$-hyperconnected. This is a contradiction. 
$(2):$ Let $f:(X, \tau, I) \rightarrow(Y, \sigma)$ be a semi- $I$-continuous function of $(X, \tau, I)$ into a Hausdorff space $(Y, \sigma)$. Since every semi- $I$-continuous function is almost $F$ - $I$-continuous, then by (1), $f$ is constant.

(3) : It follows from (2).

The implication in Theorem 20 is not reversible as shown in the following example:

Example 21. Let $X=\{a, b, c\}, \tau=\{X, \emptyset,\{a\},\{a, b\},\{a, c\}\}$ and $I=$ $\{\emptyset,\{a\}\}$. Then every semi- $I$-continuous function $f:(X, \tau, I) \rightarrow(Y, \sigma)$, where $(Y, \sigma)$ is a two point discrete space is constant but $(X, \tau, I)$ is not $\star$-hyperconnected.

Theorem 22. If $X$ is a $\star$-hyperconnected ideal space and $f:(X, \tau, I) \rightarrow$ $(Y, \sigma)$ is an almost $F$-I-continuous surjection, then $Y$ is hyperconnected.

Proof. Suppose that $Y$ is not hyperconnected. Then there exist disjoint nonempty open sets $A \subset Y$ and $B \subset Y$. Take $M=\operatorname{Int}(C l(A))$ and $N=$ $\operatorname{Int}(C l(B))$. Then $M$ and $N$ are nonempty regular open sets and $M \cap N=\emptyset$. We have $S$-I-Int $\left(f^{-1}(M)\right) \cap S-I-\operatorname{Int}\left(f^{-1}(N)\right) \subset f^{-1}(M) \cap f^{-1}(N)=\emptyset$. Since $f$ is an almost $F$ - $I$-continuous surjection, then $S$ - $I$ - $\operatorname{Int}\left(f^{-1}(M)\right) \neq \emptyset$ and $S$-I-Int $\left(f^{-1}(N)\right) \neq \emptyset$. By Lemma 7 and Theorem 11, $X$ is not $\star-$ hyperconnected. This is a contradiction.

Corollary 23. If $X$ is a $\star$-hyperconnected ideal space and $f:(X, \tau, I) \rightarrow$ $(Y, \sigma)$ is a continuous surjection, then $Y$ is hyperconnected.

Proof. Since every continuous function is almost $F$ - $I$-continuous, it follows from Theorem 22 .

Definition 24. A function $f:(X, \tau) \rightarrow(Y, \sigma, I)$ is said to be almost $F$ - $I$-open if $S$ - $I$ - $\operatorname{Int}(f(A)) \neq \emptyset$, for every nonempty regular open set $A \subset X$.

Theorem 25. If $Y$ is a $a$-hyperconnected ideal space and $f:(X, \tau) \rightarrow$ $(Y, \sigma, I)$ is an almost $F-I$-open injection, then $X$ is hyperconnected.

Proof. Let $A$ and $B$ be any nonempty open sets of $X$. Take $M=$ Int $(C l(A))$ and $N=\operatorname{Int}(C l(B))$. This implies that $M$ and $N$ are nonempty regular open sets. Since $f$ is almost $F$-I-open, then $S$-I-Int $(f(M)) \neq$ $\emptyset$ and $S$ - $I$ - $\operatorname{Int}(f(N)) \neq \emptyset$. Since $Y$ is $\star$-hyperconnected, then $\emptyset \neq S$ - $I$ $\operatorname{Int}(f(M)) \cap S$-I-Int $(f(N)) \subset f(M) \cap f(N)$. Since $f$ is an injective function, then $M \cap N \neq \emptyset$. Thus, $A \cap B \neq \emptyset$ and hence $X$ is hyperconnected. 
Corollary 26. If $Y$ is a -hyperconnected ideal space and $f:(X, \tau) \rightarrow$ $(Y, \sigma, I)$ is an open injection, then $X$ is hyperconnected.

Proof. Since every open function is almost $F$ - $I$-open, it follows from Theorem 25.

\section{REFERENCES}

1. Dontchev, J. - On pre-I-open sets and a decomposition of I-continuity, Banyan Math. J., 2 (1996).

2. Dontchev, J.; Ganster, M.; Rose, D. - Ideal resolvability, Topology Appl., 93 (1999), 1-16.

3. Hatir, E.; NoIRI, T. - On decompositions of continuity via idealization, Acta Math. Hungar., 96 (2002), 341-349.

4. Hatir, E.; Keskin, A.; Noiri, T. - On a new decomposition of continuity via idealization, JP J. Geom. Topol., 3 (2003), 53-64.

5. Hatir, E.; NoIRI, T. - On semi-I-open sets and semi-I-continuous functions, Acta Math. Hungar., 107 (2005), 345-353.

6. Janković, D.; Hamlett, T.R. - New topologies from old via ideals, Amer. Math. Monthly, 97 (1990), 295-310.

7. Kuratowski, K. - Topology, Vol. I, Academic Press, New York-London, 1966.

8. Steen, L.A.; Seebach, J.A., JR. - Counterexamples in Topology, Holt, Rinehart and Winston, Inc., New York-Montreal, Que.-London, 1970.

9. Vaidyanathaswamy, R. - The localisation theory in set-topology, Proc. Indian Acad. Sci., Sect. A., 20 (1944), 51-61.

Received: 26.XI.2009

Department of Mathematics, Canakkale Onsekiz Mart University, Terzioglu Campus, 17020 Canakkale, TURKEY eekici@comu.edu.tr

2949-1 Shiokita-cho, Hinagu, Yatsushiro-shi, Kumomoto-ken, 869-5142,

JAPAN

t.noiri@nifty.com 